\title{
ENSAMBLAJE ECOLÓGICO EN LAS LAGARTIJAS ARBÓREAS (SQUAMATA, POLYCHROTIDAE, Anolis) EN LA AMAZONÍA SUR DEL PERÚ
}

\author{
Caroll Z. Landauro
}

V. Morales ${ }^{1}$

\begin{abstract}
RESUMEN
Se analizó el ensamblaje ecológico de cuatro especies de Anolis (8 de A. fuscoauratus, 4 de $A$. bombiceps, 1 de $A$. transversalis y 3 de A. punctatus) del Parque Nacional del Manu, Perú, depositados en el Museo de Historia Natural de la URP. Se extrajo el contenido estomacal de los 16 especimenes y los ítems alimenticios fueron analizados con los Componentes Principales (CP) para establecer la correlación alimenticia de las especies de lagartijas. Con el indice de Pianka $(O)$ se estableció el grado de competencia por el alimento. Las lagartijas Anolis del Parque Nacional del Manu se caracterizan por tener hábitos alimenticios insectívoros y no mostraron competencia por el recurso, siendo principalmente estos los ácaros, neurópteros, dípteros y lepidópteros.
\end{abstract}

Palabras claves: Anolis, dieta, competencia, Manu, Perú.

\section{SUMMARY}

The ecological assembleage of four species of Anolis ( 8 of A. fuscoauratus, 4 of A. bombiceps, 1 of $A$. transversalis and 3 of $A$. punctatus) were analyzed from the National Park of the Manu, Perú; deposited in the Natural History Museum-URP. The stomach items, of 16 specimens, were analyzed with Principal Components (CP) to establish the nutritional correlation of lizard species. With the Pianka index $(O)$ was analyzed the food competition of the specimens. The Anolis lizards of the Manu National Park were insectivorous nutritional habits and they did not show competition by the resource, as acari, neuropterans mainly, dipterans and lepidopterans.

Key words: Anolis, diet, competition, Manu, Perú.

\footnotetext{
Museo de Historia Natural. Universidad Ricardo Palma. Av. Benavides 5440, Santiago de Surco, Lima 33, Perú. Apartado Postal 18-01.
} 


\section{INTRODUCCIÓN}

El género Anolis (Daudin, 1802) se caracteriza por tener un abanico gular extenso y aplanado, y las lamelas interdigitales expandidas lateralmente; este genero consta de mas de 200 especies y distribuidas desde el Sur Este de Norte América, a través de México, Centro América hasta la cuenca amazónica de Sudamérica (Ávila-Pires, 1995).

En Perú ocurren 14 especies de Anolis en el Bosque Tropical Amazónico y solamente una especie (Anolis boettgeri) en los yungas peruanos (Carrillo e Icochea, 1995). Seis especies de Anolis están presentes en la Amazonía Sur peruana, en el departamento de Madre de Dios (MD). Dentro del MD existen dos áreas protegidas de mucha importancia: El Parque Nacional del Manu y la Reserva Nacional de Tambopata. Ambas áreas naturales protegidas poseen gran diversidad de hábitat que van asociadas a la diversidad de organismos (Edwin, 1991). En el Parque Nacional del Manu se han reportado tres especies de Anolis (A. bombiceps, $A$. fuscoauratus y $A$. punctatus) en cuatro tipos de hábitat de las 9 que se has sido identificadas (Morales y McDiarmid, 1996).

El uso del hábitat de las especies de Anolis fue sugerido por Schoener
(1974) para especies del Caribe. Sin embargo, para las especies continentales es muy escasa la información ecológica para Anolis. Así, Vitt, et al. (2003) hace un estudio sobre la ecología comparativa de tres poblaciones de Anolis fuscoauratus, señalando que estas poblaciones (Ecuador y Brasil) son muy similares entre si en lo referente al uso de hábitat. Por oro lado, Vitt y Carvalho (1995) estudiaron las relaciones de nicho entre lagartijas en un hábitat de Sabana en el norte de Brasil, estos estudios asumen que las lagartijas simpátridas están separadas por el alimento, tiempo de actividad y microhábitat.

En el presente trabajo se analizó el ensamblaje de las lagartijas arbóreas en el Parque Nacional del Manu, tomando como variables la selección del hábitat y los recursos alimenticios

\section{MATERIAL Y MÉTODOS}

Se examinó el contenido estomacal de 16 especimenes de lagartijas arbóreas del genero Anolis: 8 especimenes de A. fuscoauratus, 4 de $A$. bombiceps, 1 de A. transversalis y 3 de A. puntatus, todas ellas depositadas en la colección de Herpetología del Museo de Historia Natural de la Universidad Ricardo Palma. Este material provino 
del Proyecto BIOLAT desarrollado en la Estación Pakitza ( $11^{0} 56^{\text {' }} \mathrm{S}, 71^{0} 16^{\text {' }}$ W) del Parque Nacional del Manu, Departamento de Madre de Dios, Perú. La identificación de los insectos o partes de ellos se hizo bajo el estereoscopio siguiendo la guía de Herbert (1956).

El análisis cuantitativo de los ítems consumidos por los especimenes de Anolis fue hecho con los Componentes Principales (CP) como un análisis exploratorio por las preferencias de alimentación (McGarigal, et al.2000) Con la finalidad de establecer si entre los especimenes de Anolis, del Parque Nacional del Manu, pudiera existir competencia. Se hizo el análisis de solapamiento de nichos con el programa EcoSim 7v (Gotelli y Entsminger, 2003) siguiendo el modelo de solapamiento, $\boldsymbol{O}_{i j}$, de Pianka $(1973,1986)$ :

$$
O_{i j}=\frac{\sum_{i=1}^{n} P_{i j} P_{i k}}{\sqrt{\sum_{i=1}^{n} P_{i j}^{2} \sum_{i=1}^{n} P_{i k}^{2}}}
$$

donde $p_{i j}$ y $p_{i k}$ representa la proporción de los ítems consumidos por las $j$ y $k$ especies. El valor de solapamiento varia desde cero (no existe solapamiento o no existe competencia) hasta el valor 1 (existe solapamiento o competencia).

\section{RESULTADOS}

Del análisis de la dieta de las especies de Anolis en el Parque Nacional del Manu resultaron dos Componentes Principales (CP) con el $41 \%$ de la varianza, así, el CP1 contiene el $21 \%$ de la varianza y el CP2 contiene el $20 \%$. El CP1 mostró dos variables correlativas con $x^{2}=0.511$, de índice de correlación, siendo estas los Acaris y Neurópteros. Las variables que influenciaron al CP2 fueron los Dípteros y Lepidópteros con un índice de correlación $\left(x^{2}\right)$ de 0.597 (Tabla 1). Estas variables agruparon a la mayoría de las especies de Anolis como consumidores de Acaris, Neurópteros, Dípteros y Lepidópteros (Fig. 1). Esta preferencia por las cuatro variables, arriba mencionadas, no hacen a las especies de Anolis selectivas, sino, que su espectro de dieta es mas amplia como ortópteros, coleópteros isópteros, etc. (Fig. 1) $\mathrm{y}$ eso hace que los especimenes de los Anolis sean generalistas, mostrando así, un solapamiento al azar $(O=0.091171,0.99300<p>0.00700)$ (Fig. 2). 
Tabla $N^{\circ}$ 1: Valores propios y valores de correlación de la dietas de las especies de Anolis en el Parque Nacional del Manu

\begin{tabular}{ccc}
\hline & CP1 & CP2 \\
\hline Valor propio & 2.502 & 2.373 \\
Variabilidad (\%) & 20.853 & 19.775 \\
\% acumulado & 20.853 & 40.628 \\
\hline \hline Aran & -0.136 & -0.351 \\
Coleo & 0.328 & -0.113 \\
Hyme & 0.251 & 0.016 \\
Isop & -0.053 & -0.135 \\
Orthop & 0.357 & 0.328 \\
Hemi & 0.302 & 0.007 \\
Acari & 0.511 & -0.073 \\
Diplo & -0.175 & -0.089 \\
Dipt & -0.080 & 0.597 \\
Lepido & -0.080 & 0.597 \\
Neuro & 0.511 & -0.073 \\
Semi & -0.157 & -0.085 \\
\hline
\end{tabular}

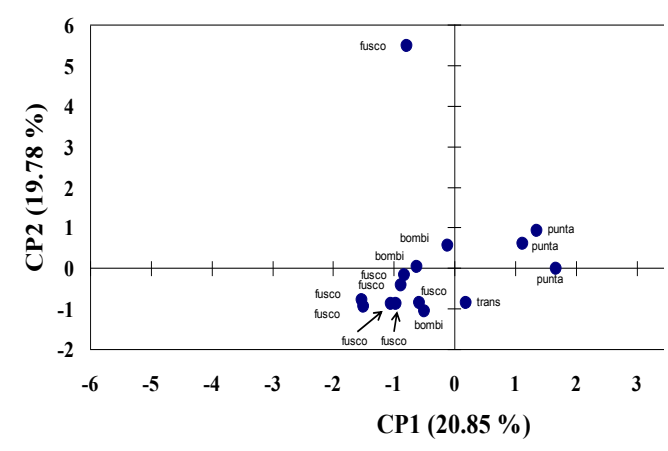

Fig. 1: Valores observados sobre la dieta de las especies de Anolis en el Parque Nacional del Manu, Perú.

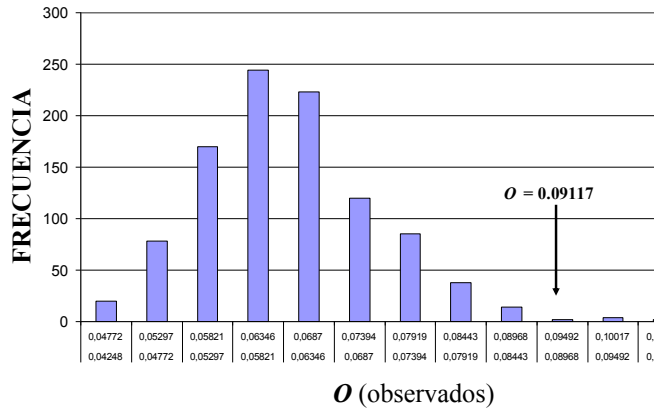

Fig 2: Biplot de los Componentes principales de la distribución de las especies de Anolis en el Parque Nacional del Manu basado en los recursos alimenticios.

\section{DISCUSIÓN}

Las Anolis en el Parque Nacional del Manu utilizaron el recurso alimenticio al azar, a pesar de compartir los mismos hábitat y microhábitats (Morales \& McDiarmid, 1996).

Vitt, et al. (2003).

La dieta de $A$. fuscoauratus en Cuyabeno (Ecuador) y Curuá-Una, Rio Juruá, Rio Ituxí, Rondônia y Rio Solimôes (Brasil) fue principalmente de arácnidos, ortópteros, larvas de insectos y coleópteros (Vitt et al., 2003). La dieta de $A$. fuscoauratus en el Parque Nacional del Manu fue principalmente correlacionada con acari y neurópteros y un individuo se correlaciono con dípteros y lepidópteros. Al parecer A. fuscoauratus es una especie de amplio espectro alimenticio y no es influenciado por las áreas geográficas. Otras especies de Anolis (A. bombiceps y A. punctatus) 
en el Parque Nacional del Manu las dietas están mas correlacionadas con acaris y neurópteros (Fig. 1). Por otro lado, Morales y McDiarmid (1996) reportaron a las tres especies de Anolis en tres tipos de hábitat haciéndolas simpátridas, pero aun así, las especies no tuvieron alto índice de competencia por al alimento y mostrando que la distribución de las lagartijas es al azar (Fig. 2).

\section{CONCLUSIONES}

Las lagartijas Anolis del Parque Nacional del Manu se caracterizan por ser típicamente arbóreas y de hábitos alimenticios insectívoros.

Las especies de Anolis en el Parque Nacional del Manu no muestran competencia por el recurso alimenticio.

La correlación alimenticia de las especies de Anolis en el Parque Nacional del Manu fue de ácaros, Neurópteros, Dípteros y Lepidópteros.

\section{LITERATURA CITADA}

AVILA-PIRES, T. C. S. 1995. Lizard of Brazilian Amazonia (Reptilia: Squamata). The Zoologische Verhandelingen Leiden, 299: 1-706. CARRILLO, N. \& ICOCHEA, J. 1995. Lista taxonómica preliminar de los reptiles vivientes del Perú. Publica- ciones del Museo de Historia Natural, Serie A Zool., 49: 1-27.

GOTELLI, N.J. \& ENTSMINGER G. L. 2003. EcoSim: Null models software for ecology. Version 7. Acquired Intelligence Inc. \& Kesey-Bear. Burlington, VT 05465. http://homepages.together. net/ gen tsmin / ecosim.htm.

MORALES, V. R. \& MCDIARMID, R. W. 1996. Annonates checklist of the amphibians and reptiles of Pakitza, Manu National Park Reserve Zone, with comments on the herpetofauna of Madre de Dios, Peru .In: Wilson, D.E and Sandoval, A (Eds), Manu: la biodiversidad del Sureste del Peru. Edit. Horizonte and Smithsonian Institution, Lima-Washington, D.C. $503-522 \mathrm{pp}$.

RODRÍGUEZ, J. A., LEAL, M. \& LOSOS, J. B. 2005. Habitat selection by the Puerto Rican yellowchinned anole, Anolis gundlachi. Canadian Journal of Zoology. 83: 983-988

PIANKA, E. R. 1973. The structure of lizard communities. Annual Review in Ecology and Systematic, 4: 53-74.

PIANKA, E. R. 1986. Ecology and natural history of desert lizards. Analyses of the ecological niche and community structure. Princeton University Press, Princeton, New Jersey, USA. 
VITT, L.J., AVILA PIRES, T.C.S., ZANI, P.A., SARTORIUS, S.S., \& ESPOSITO, M.C. 2003. Life above ground: ecology of Anolis fuscoauratus in the Amazon rain forest, and comparisons with its nearest relatives. Canadian Journal of Zoology, 81: 142-156

VITT, L.J. 1995. The Ecology of tropical lizards in the caatinga of northeast Brasil. Occasional Papers of the Oklahoma Museum of Natural History, 1: 1-29

\section{AGRADECIMIENTOS}

A mi asesor Víctor R. Morales quien es mi guía en las investigaciones. A Mercedes Gonzales, directora del Museo de Historia Natural de la Universidad Ricardo Palma, por permitirnos en utilizar el material biológico para este trabajo. 